\title{
AFERIĈ̣̃O SIMULTÂNEA DA PRESSÃo ABDOMINAL NA AVALIAÇÃO ULTRA-SONOGRÁFICA DE MULHERES COM INCONTINÊNCIA URINÁRIA DE ESFORÇO*
}

\author{
Frederico Teixeira Brandt ${ }^{1}$, Leonor Viana Nóbrega ${ }^{2}$, Carla Daisy Costa Albuquerque ${ }^{3}$, \\ Felipe Rinald Barbosa Lorenzato ${ }^{4}$, Glaucia Fonseca de Almeida ${ }^{5}$, Darla Siqueira Tenório Lima ${ }^{6}$, \\ Mariana Vila Nova de Oliveira ${ }^{7}$
}

Resumo OBJETIVO: Determinar a influência da aferição da pressão intra-abdominal na avaliação ultra-sonográfica da junção uretrovesical (JUV) e da uretra proximal (UP) em pacientes com incontinência urinária de esforço (IUE). MATERIAIS E MÉTODOS: Estudo prospectivo de corte transversal realizado na Unidade de Pesquisa em Incontinência Urinária da Universidade Federal de Pernambuco, de janeiro de 2002 a janeiro de 2005. Trinta e seis pacientes com queixas de IUE foram submetidas a ultra-sonografia perineal para avaliação da JUV e da UP com a bexiga praticamente vazia $(<50 \mathrm{ml})$, com aferição simultânea de pressão intra-abdominal. Para as avaliações, foi utilizado aparelho de ultra-som com transdutor vaginal de $7 \mathrm{MHz}$ e seletor eletrônico de mensuração de imagem real, equipado com computador e câmera fotográfica de resolução instantânea. Para a medida da pressão intra-abdominal, foi utilizado aparelho de urodinâmica com cateter de $10 \mathrm{fr}$ retal acoplado a um balão de sensor para medida da pressão intra-abdominal. RESULTADOS: As pacientes tinham idade entre 25 e 69 anos (média de 46,4 \pm 10,2 anos). À manobra de Valsalva, a pressão intra-abdominal variou entre $7 \mathrm{cmH}_{2} \mathrm{O}$ e $193 \mathrm{cmH}_{2} \mathrm{O}$ (média de 99,3 $\pm 51,8 \mathrm{cmH}_{2} \mathrm{O}$; mediana de $99,5 \mathrm{cmH}_{2} \mathrm{O}$ ). Oito das $31(25,8 \%)$ pacientes com hipermobilidade da JUV apresentaram pressão intra-abdominal inferior a $60 \mathrm{cmH}_{2} \mathrm{O}$. Não foi detectada relação estatisticamente significante entre a variação de pressão intra-abdominal e os parâmetros ultra-sonográficos em questão. CONCLUSÃO: Há um índice específico de pressão de deslocamento uretral para cada mulher com IUE. Porém, não há associação significativa entre o aumento de pressão intra-abdominal e aumento de mobilidade da JUV e UP em mulheres com quadro clínico de IUE. Unitermos: Incontinência urinária de esforço; Pressão intra-abdominal; Ultra-sonografia perineal; Junção uretrovesical.

Abstract Intraabdominal pressure measurement during ultrasound assessment of female patients with stress urinary incontinence.

OBJECTIVE: To determine the influence of intraabdominal pressure in the ultrasonographic evaluation of the urethrovesical junction (UVJ) and proximal urethra (PU) in patients with stress urinary incontinence (SUI). MATERIALS AND METHODS: A prospective, cross-sectional study was conducted at the Urinary Incontinence Research Unit of "Universidade Federal de Pernambuco", Brazil, from January 2002 to January 2005. Perineal ultrasounds of the UVJ and PU were performed in thirty-six women complaining of SUI with the bladder virtually empty $(<50 \mathrm{ml}$ ); simultaneous measurement of the intraabdominal pressure was also performed. An ultrasound machine with a computer chip and a high-resolution photographic camera connected to a $7 \mathrm{MHz}$ vaginal probe was used for the evaluation. In order to measure the intraabdominal pressure, an urodynamic equipment with a $10 \mathrm{fr}$ rectal catheter connected to a pressure sensitive balloon was used. RESULTS: The age of the patients ranged from 25 to 69 years (mean $46.4 \pm 10.2$ years). On Valsava maneuver, the intra-abdominal pressure ranged from 7 to $193 \mathrm{cmH}_{2} \mathrm{O}$ (mean: $99.3 \pm 51.8 \mathrm{cmH}_{2} \mathrm{O}$; median: $\left.99.5 \mathrm{cmH}_{2} \mathrm{O}\right)$. Eight out of $31(25.8 \%)$ patients with UVJ hypermobility had an intraabdominal pressure lower than $60 \mathrm{cmH}_{2} \mathrm{O}$. There was no statistically significant association between intraabdominal pressure and the ultrasound parameters studied. CONCLUSION: There is a specific urethral pressure index for each woman with SUI. However, there is no significant association between increase in intraabdominal pressure and increase of UVJ and UP hypermobility in women with clinical SUI.

Keywords: Stress urinary incontinence; Abdominal pressure; Perineal ultrasound; Urethrovesical junction.

* Trabalho realizado na Unidade de Pesquisa em Incontinência Urinária da Universidade Federal de Pernambuco, Recife, PE.

1. Livre-Docente, Professor Adjunto de Urologia na Universidade Federal de Pernambuco.

2. Mestre em Urologia pela Universidade Federal de Pernambuco.

3. Doutora em Urologia, Professora Adjunta de Obstetrícia na Universidade Federal de Pernambuco.
4. Doutor em Patologia Molecular, Coordenador da Disciplina de Saúde da Mulher do Mestrado em Saúde Materno-Infantil do Instituto Materno Infantil de Pernambuco.

5. Professora Assistente de Ginecologia e Obstetrícia na Universidade Federal de Pernambuco.

6. Estudante de Medicina e Bolsista em Iniciação Científica na Universidade Federal de Pernambuco.
7. Estudante de Medicina na Universidade Federal de Pernambuco.

Endereço para correspondência: Prof. Dr. Frederico Teixeira Brandt. Avenida 17 de Agosto, 2475, ap. 2801. Recife, PE, 52060-590. E-mail: fcbrandt@elogica.com.br

Recebido para publicação em 2/6/2005. Aceito, após revisão, em 29/6/2005. 


\section{INTRODUÇÃO}

Um estudo multicêntrico na França estimou em $37 \%$ a prevalência de incontinência urinária em mulheres com idade variando de 30 a 86 anos $^{(1)}$.

A incontinência urinária acomete cerca de 10 milhões de mulheres nos Estados Unidos, nas diversas faixas etárias e classes sociais, com um gasto governamental estimado em aproximadamente 20 bilhões de dólares por ano, principalmente em decorrência do emprego sistemático de estudo urodinâmico na investigação da incontinência urinária ${ }^{(2)}$.

Nas últimas décadas, a ultra-sonografia vem sendo utilizada com grande destaque na investigação da junção uretrovesical (JUV) e da uretra proximal (UP), por ser um método simples, de baixo custo, inócuo e reprodutível com facilidade. A partir de 2001, alguns autores vêm associando a ultra-sonografia da JUV ao Doppler colorido, permitindo imagens e aferições do fluxo sanguíneo ao trato urinário ${ }^{(3-8)}$.

Brandt et al. defendem o conceito de excluir-se do exame ultra-sonográfico da JUV e da UP os fatores que poderiam provocar falsa mobilidade dessas estruturas. Dessa maneira, a mobilidade da UP é avaliada de forma primária, sem a interferência de eventual contração do detrusor que determinasse de forma secundária a mobilidade da JUV e da $\mathrm{UP}^{(3)}$.

$\mathrm{Na}$ atualidade, o estudo urodinâmico continua sendo considerado padrão ouro. O exame apresenta como elemento fundamental a aferição da pressão abdominal no momento da perda urinária, com ponto de corte estimado em $60 \mathrm{cmH}_{2} \mathrm{O}^{(3)}$.

Os autores relacionam, de forma indireta, a pressão de perda com a mobilidade da JUV e da UP e inferem a respeito da resistência uretral. Classicamente, tem sido referido que a bexiga cheia e a pressão abdominal aumentada são fatores determinantes de incontinência urinária de esforço (IUE) e, por esse motivo, realizam o exame com a bexiga cheia, com a finalidade de medir a pressão abdominal no momento da perda urinária provocada pelo esforço $^{(9-15)}$.

Ainda não há referência sobre a aferição da pressão abdominal no momento da avaliação ultra-sonográfica da JUV e da
UP. Dessa forma, poderíamos estar diante de um novo e importante conceito, ao estudarmos a relação matemática e clínica entre pressão abdominal e mobilidade da JUV e da UP.

Partimos do pressuposto que a aferição da pressão abdominal na mobilidade da JUV e da UP será um passo a mais no esclarecimento da fisiopatologia da incontinência urinária e que poderá proporcionar racionalização econômica e funcional no diagnóstico e tratamento de pacientes com IUE.

Este trabalho, envolvendo mulheres com IUE, visa a estabelecer se há necessidade em aferir a pressão abdominal na avaliação ultra-sonográfica da JUV e da UP.

\section{MATERIAIS E MÉTODOS}

O protocolo de pesquisa do presente estudo foi revisado e aprovado pelo Comitê de Ética em Pesquisa envolvendo seres humanos da Universidade Federal de Pernambuco (UFPE), antes que qualquer paciente fosse convidada a participar do estudo. De janeiro de 2002 a janeiro de 2005, as mulheres com queixas de IUE atendidas na Unidade de Pesquisa em Incontinência Urinária (UPIU) da UFPE foram convidadas a participar do estudo.

Os critérios de inclusão adotados foram: idade entre 25 anos e 70 anos; presença de sintomas ou sinais de IUE; ausência de passado cirúrgico envolvendo a bexiga, a uretra ou a vagina; ausência de uretrocistocele maior que grau 2.

Os critérios de exclusão foram: diagnóstico de bexiga neurogênica firmado antes ou durante a pesquisa; queixa sugestiva de infecção urinária no dia da realização do estudo ultra-sonográfico.

\section{Caracterização da amostra}

Foram investigadas 36 mulheres referindo queixas de IUE, com idades variando entre 25 e 69 anos, cuja média foi de $46,4 \pm 10,2$ anos e a mediana foi de 46,5 anos. Identificou-se que $75 \%$ das mulheres tinham 40 anos ou mais.

Para a realização dos exames foi utilizado um aparelho de ultra-som da marca Aloka SSD500, acoplado a transdutor vaginal de $7 \mathrm{MHz}$ e seletor eletrônico de mensuração de imagem real, equipado com computador e câmera fotográfica de resolução instantânea.

Era solicitado que a paciente esvaziasse a bexiga espontaneamente, um pouco antes do exame, e se mantivesse em posição de litotomia dorsal, com as pernas fletidas, como descrito por Brandt et al..$^{(3,4)}$.

Após cateterismo retal para aferição da pressão abdominal pelo aparelho de urodinâmica, colocou-se o transdutor, revestido por preservativo e lubrificado com solução gelatinosa de contato, tocando a vulva, numa localização em que o ultrasonografista podia identificar a uretra, a bexiga, o colo vesical e a sínfise púbica, estruturas com ecotexturas características.

Para a medida da pressão abdominal, foi utilizado aparelho de urodinâmica Uromaster MPX616, da Dynamed, que mediu apenas a pressão intra-abdominal durante a manobra de Valsalva, através de um cateter de $10 \mathrm{fr}$ retal, com balão para medida da pressão abdominal (Figura 1). Todos os exames - uroginecológico, medida da pressão abdominal e ultra-sonográfico foram realizados pelos autores do trabalho.

Durante o exame foi aferida a pressão abdominal no momento da manobra de Valsalva, por meio de transdutor retal, do que se originou registro gráfico (Figura 1).

\section{RESULTADOS}

À manobra de Valsalva, a pressão intra-abdominal variou entre $7 \mathrm{cmH}_{2} \mathrm{O}$ e 193 $\mathrm{cmH}_{2} \mathrm{O}$, sendo $99,3 \pm 51,8 \mathrm{cmH}_{2} \mathrm{O}$ a média com o desvio-padrão e $99,5 \mathrm{cmH}_{2} \mathrm{O}$ a mediana. Oito das $31(25,8 \%)$ pacientes com hipermobilidade da JUV apresentaram pressão intra-abdominal inferior a 60 $\mathrm{cmH}_{2} \mathrm{O}$.

A Figura 2 mostra que quanto maior foi a pressão intra-abdominal durante a avaliação ultra-sonográfica perineal da JUV, menor foi o número de mulheres que apresentavam deslocamento vertical acima de $9 \mathrm{~mm}$ (hipermobilidade).

Foi realizado o teste de Kruskal-Wallis, correspondente à análise de variância para amostras sem distribuição normal, para verificar a relação entre a variação de pressão abdominal e de cada um dos parâmetros ultra-sonográficos, não tendo sido detectada significância estatística nessas relações, conforme indicado na Tabela 1. 


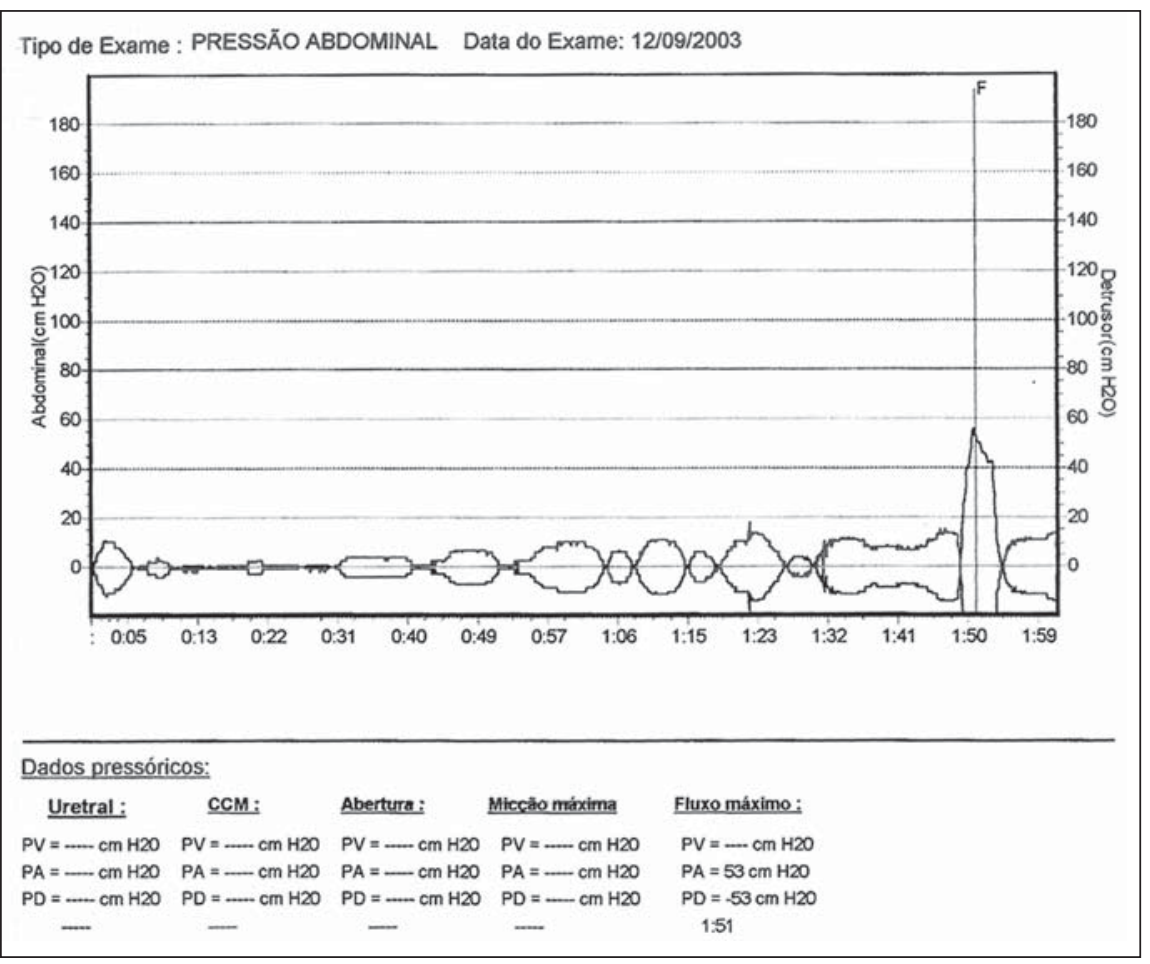

Figura 1. Registro gráfico da medida da pressão intra-abdominal de uma paciente com IUE clínica, avaliada na UPIU-UFPE.

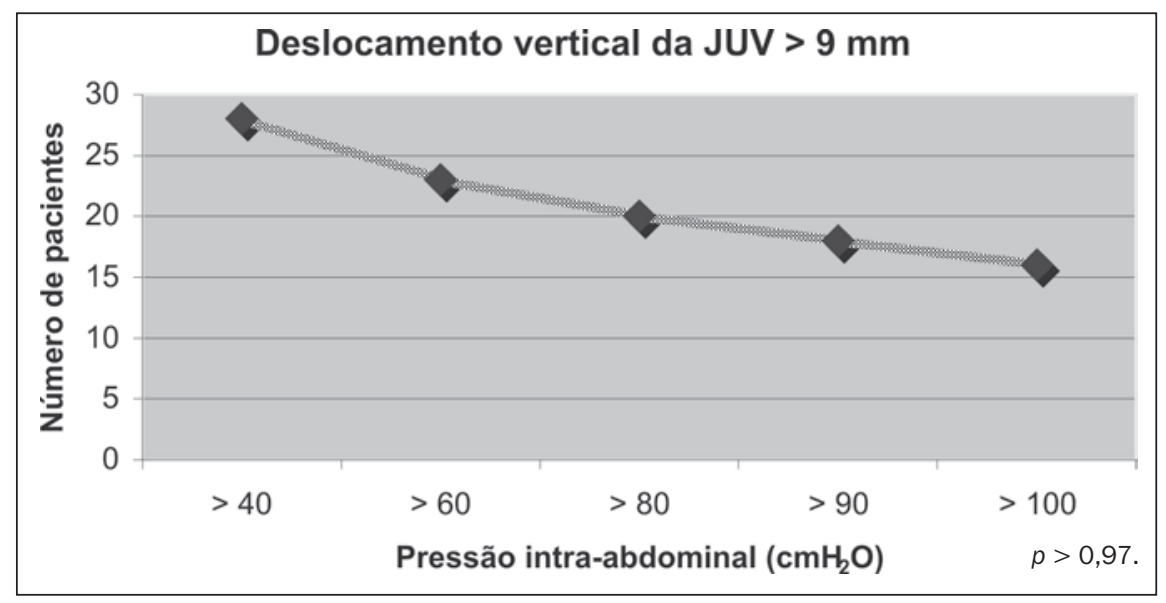

Figura 2. Comportamento da hipermobilidade da JUV em relação ao aumento gradativo da pressão intraabdominal.

É demonstrado, de forma não estratificada, uma tendência geral de hipermobilidade vertical da JUV com uma pressão intra-abdominal maior que $60 \mathrm{cmH}_{2} \mathrm{O}$, conforme indicado na Tabela 2.

\section{DISCUSSÃO}

A IUE se reveste de grande importância não só como doença, mas, principalmente, pelas repercussões sociais e pela
Tabela 1 Parâmetros estatísticos do teste de Kruskal-Wallis relacionando pressão intra-abdominal e medidas ultra-sonográficas. (UPIU-UFPE, janeiro 2002 a janeiro 2005.)

\begin{tabular}{|l|r|c|}
\hline $\begin{array}{l}\text { Parâmetro da ultra- } \\
\text { sonografia perineal }\end{array}$ & \multicolumn{1}{|c|}{$\chi^{2}$} & $p$ \\
\hline DPU & 34,67 & 0,44 \\
UP & 35,00 & 0,42 \\
HJUV & 8,62 & 0,26 \\
VJUV & 0,79 & 0,73 \\
Deslocamento & 0,92 & 0,70 \\
\hline
\end{tabular}

DPU, distância pubouretral; UP, uretra proximal; HJUV horizontal da junção uretrovesical; VJUV, vertical da junção uretrovesical.

Tabela 2 Distribuição das pacientes com IUE que apresentaram hipermobilidade da JUV (deslocamento vertical $>9 \mathrm{~mm}$ ) quando a pressão intra-abdominal excedia $60 \mathrm{cmH}_{2} \mathrm{O}$ durante a avaliação ultrasonográfica perineal. (UPIU-UFPE, janeiro 2002 a janeiro 2005.)

\begin{tabular}{|l|c|c|c|}
\hline \multirow{2}{*}{$\begin{array}{l}\text { Pressão intra- } \\
\text { abdominal }\end{array}$} & \multicolumn{2}{|c|}{$\begin{array}{c}\text { Deslocamento vertical } \\
\text { da JUV }\end{array}$} & \\
\cline { 2 - 4 } & $>9 \mathrm{~mm}$ & $\leq 9 \mathrm{~mm}$ & Total \\
\hline$>60 \mathrm{cmH}_{2} \mathrm{O}$ & 23 & 3 & 26 \\
$\leq 60 \mathrm{cmH}_{2} \mathrm{O}$ & 8 & 2 & 10 \\
\hline Total & 31 & 5 & 36 \\
\hline
\end{tabular}

JUV, junção uretrovesical. Risco relativo $=1,11 ; \chi^{2}=$ 0,$01 ; p=0,60$.

tudo utilizando o exame ultra-sonográfico da JUV e da UP, por ser simples, de baixo custo, fisiológico e reprodutível.

A intenção deste trabalho não é se contrapor formalmente à indicação de estudo urodinâmico, completo ou simplificado, na investigação de pacientes com IUE. Pelo contrário, é realçar que ele é o padrão ouro na investigação de pacientes com IUE. Mas, é necessário admitir que existam interpretações diferentes e contraditórias em relação a esse tema.

Por outro lado, para efeito de análise, vale ressaltar que a urodinâmica historicamente tem apresentado várias falhas que foram reconhecidas e desmistificadas com o tempo, por exemplo, a exagerada importância dada aos valores de pressão uretral.

$\mathrm{O}$ destaque atual da urodinâmica se prende fundamentalmente à pressão de perda. Esta medida é utilizada para definir, de forma indireta, o tipo de mobilidade da uretra. Já a ultra-sonografia, por ser um método que não induz a contração do detrusor, avalia de forma direta a mobilidade 
vertical e horizontal da JUV e da UP, além do que é um exame com imagem em tempo real e mais cômodo ${ }^{(\mathbf{3 , 4})}$.

Brandt $^{(3)}$ considera que a urodinâmica não faz diagnóstico concreto de IUE, tanto que não existe trabalho, utilizando o método, mostrando um padrão compatível com o pré e o pós-operatório de pacientes com IUE. Porém, é consenso que o exame diagnostica situações associadas importantes para o tratamento ${ }^{(\mathbf{9 - 1 5})}$. A ultra-sonografia também não faz diagnóstico de IUE, mas define com clareza os parâmetros relacionados à mobilidade da JUV e da UP, antes e após o tratamento da $\operatorname{IUE}^{(\mathbf{3 , 4})}$.

É freqüentemente citado que há necessidade de determinar se as pacientes com IUE têm, também, contração do detrusor diagnosticada pela urodinâmica ${ }^{(9-15)}$. Mas, temos discordado dessa interpretação. Primeiro, porque cerca de $50 \%$ das pacientes com IUE têm contração involuntária do detrusor decorrente da hipermobilidade uretral e que desaparece com o tratamento cirúrgico adequado. Segundo, pode ser acrescentado que uma paciente com IUE clínica, associada à contração involuntária do detrusor, e hipermobilidade primária da uretra tem indicação de correção cirúrgica da mobilidade anormal, mesmo que permaneça no pós-operatório com contração involuntária do detrusor. Nessa hipótese, a contração involuntária do detrusor deve ser tratada de forma independente da queixa de IUE ${ }^{(3,4)}$.

A inclusão das pacientes baseou-se nos sintomas, conceituados conforme Abrams et al., já que seriam feitas a investigação de incontinência urinária e a conseqüente determinação da presença de hipermobilidade da JUV, critério para diagnóstico e decisão terapêutica ${ }^{(19)}$.

Existe discussão antiga, cada dia mais interessante, a respeito da importância da mobilidade da JUV e da UP na etiopatogenia multifatorial da IUE, inclusive se a referida mobilidade está diretamente relacionada ao gradiente variável da pressão abdominal $^{(\mathbf{1 7}, 18)}$. Como é consenso que cada paciente executa uma pressão de Valsalva diferente no momento do exame, no nosso entendimento é importante e válida essa pesquisa que busca aferir de forma simultânea o aumento de pressão abdominal e mobilidade da JUV e da UP.
O que se sabe da literatura é que existem parâmetros variáveis de pressão abdominal, pressão vesical e mobilidade uretral em cada paciente com IUE e em cada momento do exame ${ }^{(\mathbf{9 - 1 5})}$.

A hipótese inicial deste estudo seria demonstrar que a partir da medida da pressão abdominal de forma simultânea com a ultra-sonografia da JUV e da UP, poderia ser estabelecida uma constante pressórica para o deslocamento uretral. Cada paciente teria uma pressão de deslocamento uretral real, que associada à história clínica de IUE poderia orientar de forma objetiva na conduta terapêutica. Entretanto, como nossos dados demonstram não haver influência significativa da pressão sobre os parâmetros de estudados, esta hipótese está descartada.

O fator causal das modificações das medidas entre a situação de repouso e a de esforço foi o aumento da pressão intra-abdominal obtido por meio da manobra de Valsalva. Isto não significa dizer que a IUE dessas pacientes teve como causa, exclusiva, o aumento da pressão intra-abdominal, até mesmo porque o estudo em pauta não teve esse objetivo, assim como é conceitual que a IUE tem mecanismos multifatoriais $^{(\mathbf{1 7}, 18)}$.

Entretanto, como cada paciente foi seu padrão, o exame foi realizado com a bexiga quase vazia e o único fator que se alterou foi a pressão intra-abdominal, com a manobra de Valsalva, pode-se supor a existência de uma relação causal entre a mobilidade da JUV e da UP e o aumento da pressão intra-abdominal, ao menos com pressões relativamente baixas, entre 40 e 60 $\mathrm{cmH}_{2} \mathrm{O}$. Tanto assim é, que oito $(25,8 \%)$ das 31 pacientes que apresentaram hipermobilidade vertical da JUV a fizeram com pressão intra-abdominal inferior a 60 $\mathrm{cmH}_{2} \mathrm{O}$ (Tabela 2), e 23 (74,2\%) apresentaram hipermobilidade com pressão intraabdominal superior a $60 \mathrm{cmH}_{2} \mathrm{O}$, em similaridade com o conceito de pressão de perda uretral ${ }^{(\mathbf{9 , 1 0})}$. Com esses dados podese sugerir que é dispensável que a paciente faça um grande esforço abdominal, por exemplo, tossir no momento do exame, sendo necessário, apenas, que a paciente execute a manobra habitual de Valsalva.

Pode-se afirmar que a pressão abdominal não é um parâmetro isolado para alte- ração das medidas ultra-sonográficas, o que concorda com os achados de outros autores, que afirmam que a pressão abdominal atua como um dos fatores a provocar IUE, mas não o único ${ }^{(11,17,20)}$.

Nosso estudo ultra-sonográfico é similar ao de Madjar et al. ${ }^{(\mathbf{1 0})}$, que, ao analisarem a relação entre variação de pressão abdominal e a pressão de perda sob esforço, por meio de estudo urodinâmico, concluíram que o valor basal da pressão abdominal varia muito de paciente para paciente, na dependência da composição pondo-estatural e do hábito do paciente em suportar pressão abdominal elevada. Por outro lado, reforça a teoria desta pesquisa, que busca o conceito de pressão de deslocamento uretral, um índice específico para cada paciente, que poderá servir como um parâmetro a mais na orientação terapêutica para IUE, independentemente da hipótese de causa multifatorial.

Este trabalho demonstra que não há relação exclusiva entre o aumento de pressão intra-abdominal e os deslocamentos da JUV e da UP e que há um índice específico de pressão de deslocamento uretral da paciente, indicando a necessidade de se ampliar a investigação, ora apresentada, para poder-se esclarecer adequadamente que fatores atuam na etiopatogenia da IUE. Demonstra, também, que do ponto de vista prático cada paciente com IUE apresenta o maior percentual de deslocamento com uma pressão abdominal abaixo de $40 \mathrm{cmH}_{2} \mathrm{O}$, compatível com a manobra de Valsalva, e que é desnecessário aferir a pressão abdominal nos exames ultra-sonográficos de rotina para investigação da JUV e da UP.

\section{CONCLUSÃO}

Há um índice específico de pressão de deslocamento uretral para cada paciente. Entretanto, não há associação significativa entre o aumento de pressão intra-abdominal e o aumento de deslocamento da JUV e da UP em pacientes com quadro clínico de IUE.

\section{REFERÊNCIAS}

1. Minaire P, Jacquetin B. La prévalence de l'incontinence urinaire féminine en médicine générale. J Gynecol Obstet Biol Reprod 1992;21:731-738.

2. Johnson VY. Bladder neck suspension nursing care: 
preop, postop, and beyond. Perspectives in Nursing Strategies 2002;8:53-57.

3. Brandt FT. Estudo dos parâmetros ultra-sonográficos da uretra proximal e da mobilidade da junção uretrovesical em mulheres, visando estabelecer a importância clínica. (Tese de Livre-Docência). São Paulo: USP, 1999.

4. Brandt FT, Albuquerque CDC, Arraes AF, Albuquerque GF, Barbosa CD, Araújo CM. Influência do volume vesical na avaliação ultra-sonográfica da junção uretrovesical e uretra proximal. Radiol Bras 2005;38:33-36.

5. Sandvik H. Health information and interaction on the internet: a survey of female urinary incontinence. BMJ 1999;319:29-32.

6. Brandt FT, Albuquerque CDC, Lorenzato FR, Amaral FJ. Perineal assessment of urethrovesical junction mobility in young continent females. Int Urogynecol J Pelvic Floor Dysfunct 2000;11:18 22.

7. Dietz HP, Clarke B. The influence of posture on perineal ultrasound imaging parameters. Int Urogynecol J Pelvic Floor Dysfunct 2001;12:104106.
8. Dietz HP, Clarke B. Translabial color Doppler urodynamics. Int Urogynecol J Pelvic Floor Dysfunct 2001;12:304-307.

9. Koelbl H, Bernaschek G, Deutinger J. Assessment of female urinary incontinence by introital sonography. J Clin Ultrasound 1990;18:370-374.

10. Madjar S, Balzarro M, Appell RA, Tchegen MB, Nelson D. Baseline abdominal pressure and Valsalva leak point pressures-correlation with clinical and urodynamic data. Neurourol Urodyn 2003;22: 2-6.

11. Howard D, Miller JM, Delancey JO, Ashton-Miller JA. Differential effects of cough, Valsalva, and continence status on vesical neck movement. Obstet Gynecol 2000;95:535-540.

12. Fusco MA, Martin RS, Chang MC. Estimation of intra-abdominal pressure by bladder pressure measurement: validity and methodology. J Trauma 2001;50:297-302.

13. McIntosh SL, Griffiths CJ, Drinnan MJ, Robson WA, Ramsden PD, Pickard RS. Noninvasive measurement of bladder pressure. Does mechanical interruption of the urinary stream inhibit detrusor contraction? J Urol 2003;169:1003-1006.
14. Sultana CJ. Urethral closure pressure and leak-point pressure in incontinent women. Obstet Gynecol 1995;86:839-842.

15. Swift SE, Ostergard DR. A comparison of stress leak-point pressure and maximal urethral closure pressure in patients with genuine stress incontinence. Obstet Gynecol 1995;85(5 Pt 1):704-708.

16. Gray M. Stress urinary incontinence in women. J Am Acad Nurse Pract 2004;16:188-197.

17. Ulmsten U. Some reflections and hypotheses on the pathophysiology of female urinary incontinence. Acta Obstet Gynecol Scand Suppl 1997;166:3-8.

18. Mouritsen L, Lose G, Ulmsten U, et al. Consensus of basic assessment of female incontinence. Acta Obstet Gynecol Scand Suppl 1997;166:59-60.

19. Abrams P, Cardozo L, Fall M, et al. The standardisation of terminology of lower urinary tract function: report from the Standardisation Sub-Committee of the International Continence Society. Neurourol Urodyn 2002;21:1676-1678.

20. Culligan PJ, Heit M. Urinary incontinence in women: evaluation and management. Am Fam Physician 2000;62:2433-44, 2447, 2452. 